\title{
Stability of transverse dipole modes in coasting ion beams with nonlinear space charge, octupoles, and chromaticity
}

\author{
V. Kornilov, O. Boine-Frankenheim, and I. Hofmann \\ Gesellschaft für Schwerionenforschung mbH, Darmstadt, Planckstrasse 1, 64291 Darmstadt, Germany
}

(Received 16 July 2007; published 8 January 2008)

\begin{abstract}
The influence of the nonlinear space-charge force, which causes an amplitude-dependent tune shift, on the transverse stability of a coasting beam with impedances is investigated. In particular, the interplay of nonlinear space charge with a cubic lattice nonlinearity (octupoles) and with linear chromatic effects is considered. For each case, the stability diagram obtained from a dispersion relation with two-dimensional tune spread is compared with results from a simulation scan. For the latter, a 3D particle tracking code is used, with self-consistent 2D space charge, coupling to transverse impedances, chromaticity, and nonlinear lattice effects. The validity of non-self-consistent space-charge models in analytical and in numerical approaches is examined. We reconfirm that, although nonlinear space charge does not provide Landau damping of its own, it modifies strongly the stability properties and must be taken into account in an analysis of Landau damping induced by external (octupole or chromaticity) effects. It is demonstrated that non-self-consistent approaches for space charge are not always applicable.
\end{abstract}

DOI: 10.1103/PhysRevSTAB.11.014201

PACS numbers: 29.27.Bd

\section{INTRODUCTION}

Space-charge effects play an important role in many existing and future high intensity ring accelerators, especially at injection energies or close to transition. Control of space charge is crucial for the envisaged operation with high quality and high intensity beams in the FAIR synchrotrons [1]. It is well known that the transverse space-charge force can modify instability thresholds and growth rates of coherent instabilities driven by ring impedances. In order to accurately predict impedance budgets for high current machines, like the FAIR synchrotrons, a detailed understanding of the interplay of space charge, nonlinear focusing fields, and impedances is required. In this work, we focus on the transverse dipole instability of a coasting beam induced by ring impedances.

Landau damping due to the finite momentum spread and chromaticity is a stabilizing mechanism of transverse dipole modes. However, the space-charge tune shift can cause the "loss of Landau damping" (see, e.g., Refs. [2,3]) for transverse dipole oscillations. Additionally, imaginary coupling impedances can shift the coherent frequency and thus affect transverse beam stability. For example, especially at injection energies in heavy ion synchrotrons, the image charge force shifts the dipole frequency and thus compensates part of the direct space-charge tune shift. It is then necessary to analyze beam stability for different possible impedance spectra, especially for accelerators with multiple beam scenarios.

A feedback system is a potential cure in the general case. Alternatively octupoles can be installed for increased Landau damping due to an amplitude-dependent tune shift. Octupoles are routinely used in many existing accelerators; they have been successfully used to cure transverse instabilities in the PS booster at CERN [4]. For accurate pre- dictions of stability threshold, the effect of the spacecharge nonlinearity - i.e., the amplitude-dependent incoherent tune shift due to nonhomogenous transverse beam profile-can become important, but has been addressed only in a few works so far.

One of the first attempts to describe the influence of nonlinear space charge was made in Refs. [4,5] with a dispersion relation based on a heuristic derivation. It was concluded that nonlinear space charge alone does not provide damping, but combined with an external nonlinearity (e.g., an octupole) it results in a significantly larger stability area than that due to the octupole alone. It was also shown that for one of the octupole polarities the stability enhancement is stronger than for the other polarity. This model was later used for the LHC in [6,7] and has been extended in [8], where tune shifts due to momentum spread and chromaticity have been included. The transverse stability for the combination of nonlinear space charge with octupoles has been also studied using more elaborate analytic models in Refs. $[9,10]$. The modification of the stability boundary due to nonlinear space charge was confirmed in these studies.

However, there has been still some uncertainty about the role of nonlinear space charge. Some authors (e.g., [11]) argue that the nonlinearity in the space-charge force should not have an effect and assume betatron oscillations to be linear in their stability analysis. The predictions of the dispersion relations mentioned above have not been directly compared with experiments, computer simulations, or with each other.

To resolve these uncertainties we perform particle-incell (PIC) simulations with the code PATRIC [12] for several types of combinations of nonlinear space charge with other damping mechanisms. In parallel, we solve the dispersion relation from Ref. [8] in an approximate manner and use it 
as a guide to understand physical effects and to choose the most interesting cases for direct comparisons. Twodimensional betatron tune spreads are taken into account. In particular, we study the case of strong space charge and weak octupoles, as it resembles the situation in the FAIR synchrotrons. We also address the question about the applicability of non-self-consistent models for space charge in numerical simulations. The corresponding simulation runs also provide us interesting insights into the interplay of space-charge effects.

In the next chapter, the basic concepts and notation of the present work are explained. In Sec. III, the particle tracking code PATRIC and methods of simulations scans are presented, while Sec. IV is devoted to the dispersion relation. Results of numerical simulations and dispersion relation solutions are compared in Sec. V. First, the case without external effects is considered. In Sec. VB, the combination of nonlinear space charge with octupole nonlinearity is discussed. The corresponding simulations with non-self-consistent space charge are presented in Sec. V C. Simulations and dispersion relation solutions for the transverse Gaussian distribution are compared in Sec. V D, both for self-consistent and non-self-consistent space charge. Section VE is devoted to the combination of nonlinear space charge with tune shifts due to chromaticity and momentum spread. The results are discussed and summarized in the Conclusions.

\section{BASIC DEFINITIONS}

The object of our investigation is a coasting beam and the following characteristics are of importance for this work. $Q$ denotes the particle betatron tune and we use $\Delta Q$ to denote tune shifts with respect to the bare betatron tune. $\Delta Q_{\text {coh }}$ is the coherent tune shift which is induced by an impedance $Z^{\perp}(\Omega)$, where $\Omega$ is the coherent frequency which we observe. Both $\Delta Q_{\text {coh }}$ and $\Omega$ are complex values. Note that under the impedance $Z^{\perp}$ we understand only the interaction with the beam surroundings which does not include, e.g., the direct space-charge force. The real coherent tune shift in the absence of damping mechanisms can be calculated as

$$
\operatorname{Re}\left(\Delta Q_{\text {coh }}\right)=\mathcal{D} \cdot \operatorname{Im}\left(Z^{\perp}\right) \quad \text { with } \mathcal{D}=\frac{N q^{2}}{4 \pi m \gamma C \omega_{\beta 0}}
$$

where $N$ is the number of ions, $q$ is the particle charge, $m$ is the ion mass, $\gamma$ is the relativistic parameter, $C$ is the ring circumference, and $\omega_{\beta 0}$ is the bare betatron frequency. With $\Delta Q_{\text {inc }}$ we denote the incoherent tune shift induced by space charge, which is a real value. For a beam with the $\mathrm{KV}$ (Kapchinsky-Vladimirsky) distribution and radius $r_{\mathrm{kv}}$, the shift in the betatron tune $\Delta Q_{\mathrm{kv}}$ due to direct space charge is identical for all the particles and is given by

$$
\Delta Q_{\mathrm{kv}}=-\frac{4 \pi}{\left(r_{\mathrm{kv}} \gamma\right)^{2} \omega_{0}} \mathcal{D}
$$

This tune shift is called linear space charge, $\omega_{0}$ is the revolution frequency. Generally, space charge causes different tune shifts depending on amplitudes of the incoherent motion,

$$
\Delta Q_{\mathrm{inc}}(a, b)=\Delta Q_{\mathrm{kv}}[1+\kappa(a, b)],
$$

where $a$ and $b$ are amplitudes of the individual oscillations in the horizontal and vertical plane, respectively. The function $\kappa(a, b)$ depends on the transverse beam distribution.

Throughout this work, we use a normalized impedance in the form

$$
V+i U=\frac{\mathcal{D}}{\left|\Delta Q_{\mathrm{kv}}\right|} Z^{\perp},
$$

which means that $V$ is proportional to the resistive impedance, $U$ is proportional to $\operatorname{Im}\left(Z^{\perp}\right)$ and to the coherent tune shift. This normalization implies that the coherent tune shift causes a shift $\Delta U=\operatorname{Re}\left(\Delta Q_{\text {coh }}\right) /\left|\Delta Q_{\mathrm{kv}}\right|$. The incoherent tune shift of linear space charge corresponds then to $\Delta U=-1$.

A momentum shift $\Delta p$ together with the chromaticity $\xi$ causes an incoherent tune shift $\Delta Q_{\xi}=\xi Q_{0} \Delta p / p_{0}$; the corresponding tune spread provides a damping mechanism which we refer to as linear Landau damping in the contest of the present study.

External nonlinearities also induce amplitude-dependent incoherent tune shifts. We model an external nonlinearity with the cubic component of the octupole magnetic field,

$$
\begin{gathered}
B_{x}=-K_{3} \frac{B \rho}{6} y^{3}, \quad B_{y}=K_{3} \frac{B \rho}{6} x^{3}, \\
\text { with } K_{3}=\frac{1}{B \rho} \frac{\mathrm{d}^{3} B_{y}}{\mathrm{~d} x^{3}} .
\end{gathered}
$$

These incoherent tune shifts are different for each individual particle. Hence, there are characteristic tune spreads which we denote as $\delta Q_{\text {sc }}$ for the space-charge tune spread, $\delta Q_{\text {oct }}$ for that induced by the octupole nonlinearity, and $\delta Q_{\xi}$ for the tune spread due to chromaticity. The spacecharge tune spread $\delta Q_{\mathrm{sc}}$ depends on the space-charge tune shift and on the type of transverse distribution, for example, for a Gaussian profile it is close to $2 \Delta Q_{\mathrm{KV}}$. To indicate the strength of the octupole nonlinearity in comparison with nonlinear space charge, we use the parameter $\chi_{\mathrm{oct}}=\delta Q_{\mathrm{oct}} / \delta Q_{\mathrm{sc}}$, and for the chromaticity effect we use $\chi_{\xi}=\delta Q_{\xi} / \delta Q_{\mathrm{sc}}$.

It is an intrinsic property of space charge that for any nonhollow distribution the nonlinear tune shift decreases in the absolute value with increasing amplitude. The cubic component of the octupole magnetic field from Eq. (5) causes an incoherent tune shift, which grows in the absolute value with amplitude $\Delta Q_{\text {oct }}(a) \propto-K_{3} a^{2}$. Since space 
charge reduces the tune shift, there is a certain octupole polarity (in this case a negative $K_{3}$ ) which enhances the effect of nonlinear space charge and is referred to here as the advantageous polarity. The opposite case we denote as the disadvantageous polarity.

\section{PARTICLE-IN-CELL SIMULATION MODEL}

The particle-in-cell tracking code PATRIC [12] has been optimized for 3D studies of transverse coherent instabilities in coasting and bunched beams.

In the present version of the code the space-charge field can be calculated using a self-consistent model or a "frozen" model. In the self-consistent model the Poisson equation is solved exactly for the disturbed particle distribution. The latter implies electric fields which keep the configuration regardless of the modifications in particle distribution, but follows the beam center. For bunched beams, one can use a multiple-slice approach in order to resolve 3D spacecharge effects, but in this work we consider coasting beams and use the 2D approach. For self-consistent space charge, two solvers of the Poisson equation are implemented, for rectangular and elliptic boundary conditions with arbitrary side ratio. The solver for elliptic boundaries is based on the fast direct solver in a 2D elliptical domain [13]. For frozen space charge, two options are implemented. In the case of the waterbag distribution the space-charge field can be calculated analytically and the resulting force is used directly in the code. For other distributions (like Gaussian), the field configuration is obtained at the first time step and is then shifted with $(\bar{x}, \bar{y})$ during the rest of the run. Note that under a waterbag distribution for the transverse beam profile we understand a distribution in $4 \mathrm{D}$ phase space, which results in a parabolic-type density distribution in $x-y$ space.

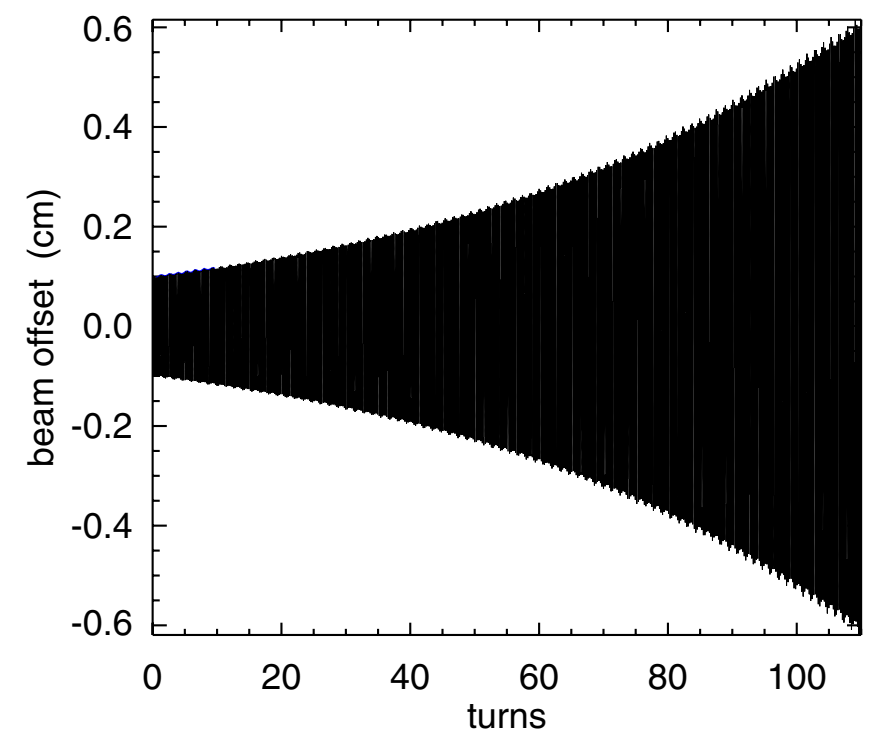

The transverse impedance implementation allows one to model arbitrary impedance spectra. Details of the implementation can be found in Ref. [12]. For coasting beams and coherent oscillations with $\left|\Delta Q_{\text {coh }}\right| \ll Q_{0}$ and $\operatorname{Re}\left(\Delta Q_{\text {coh }}\right) \gg \operatorname{Im}\left(\Delta Q_{\text {coh }}\right)$, the horizontal impedance kick per turn can be formulated as

$$
\begin{aligned}
\Delta x^{\prime}(t)= & \frac{N q^{2}}{p_{0} C}\left[-\bar{x}(t) \operatorname{Im}\left(Z^{\perp}\right)+\frac{\mathrm{d} \bar{x}}{\mathrm{~d} t}(t)\right. \\
& \left.\times \frac{\operatorname{Re}\left(Z^{\perp}\right)}{\omega_{\beta 0}+\operatorname{Re}\left(\Delta Q_{\mathrm{coh}}\right) \omega_{0}}\right],
\end{aligned}
$$

where $\bar{x}$ is the beam mass center in the horizontal plane and $Z^{\perp}$ is a transverse impedance acting on a coasting beam. Since in our $2 \mathrm{D}$ problem $Z^{\perp}$ is not a spectrum but a value, that corresponds to the impedance at the frequency of the slow wave $\left(n \omega_{0}-\omega_{\beta}\right)$ for the most unstable mode. By varying the mode number $n$ and the corresponding parameters (e.g., the chromatic tune spread), this model can be applied to an arbitrary betatron frequency. The aim of our simulation studies is to obtain the stability boundary in the complex $Z^{\perp}$ impedance plan. Hence, series of simulations have been performed with varying both $\operatorname{Re}\left(Z^{\perp}\right)$ and $\operatorname{Im}\left(Z^{\perp}\right)$, each pair corresponding to certain $U, V$. As a result, a direct comparison of PIC simulations with a dispersion relation can be conducted. A conducting wall is assumed as a boundary condition for self-consistent simulations. Thus, for the shift of the coherent frequency the effect of the image charges additionally to $\operatorname{Im}\left(Z^{\perp}\right)$ in Eq. (6) must be taken into account. Chromatic effects and a cubic nonlinearity are implemented as transverse momentum kicks, uniformly distributed over the ring.

The simulation model was chosen in a way to resemble the beam physics model underlying the dispersion relation. The rms envelope of the beam was matched; a uniform

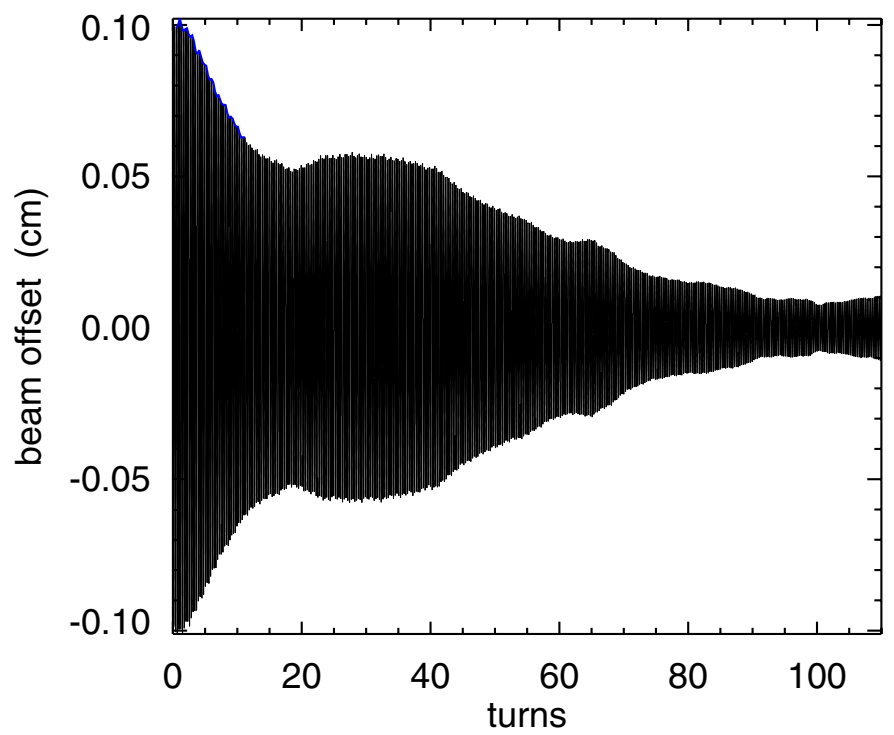

FIG. 1. Representative beam center oscillations from PATRIC simulations for two different $\operatorname{Im}\left(Z^{\perp}\right)$ and fixed $\operatorname{Re}\left(Z^{\perp}\right)$; left: instability; right: damping dominates (stable). Beam conditions correspond to Fig. 4, left. 
focusing model was used for the lattice. Both for the simulations and for the solution of the dispersion relation, round beams in transverse plane were assumed, equal tunes and chromaticities for both transverse planes were taken. Impedances in the horizontal plane were applied and therefore dipole oscillations in the horizontal plane are excited. As the initial transverse distribution, we use waterbag and Gaussian distributions. For the longitudinal momentum, a Gaussian distribution was assumed. Coasting beam parameters similar to the ones foreseen in the SIS 18 [1] have been assumed, with the factor 5 larger intensity to make growth times smaller for reasonable computing times. The other parameters (momentum spreads, etc.) were then scaled to achieve the same normalized impedances $U, V$ and thus make sure that the same stability behavior results.

An example for our simulation scans is presented in Fig. 1 with two typical time evolutions of the beam center, one of them is an unstable situation and another beam is stabilized by damping. In both cases, the beam oscillates horizontally in a rigid dipole mode. The instability from the left-hand side of Fig. 1 evolves with the growth time $0.26 \mathrm{~ms}$, which corresponds to 55.5 turns. These simulations are made for the case of the combination of an octupole and nonlinear space charge, corresponding results are presented in Fig. 4, left, and discussed below. For both runs the same $\operatorname{Re}\left(Z^{\perp}\right)$ has been applied, but the coherent line shift which is governed by $\operatorname{Im}\left(Z^{\perp}\right)$ is different. The simulation run in Fig. 1, left, corresponds to the normalized impedance $V=3.6 \times 10^{-3}, U=-0.1$; the one in Fig. 1, right, corresponds to $V=3.6 \times 10^{-3}, U=-0.9$ (see Fig. 4, left). Without incoherent tune spread due to an octupole, the perturbation would grow equally for both of these beams.

\section{DISPERSION RELATION}

By following closely the approach given in Ref. [8], the dispersion relation for the coherent mode frequency $\Omega$ in the horizontal plane is constructed,

$$
\begin{aligned}
\int \frac{\Delta Q_{\mathrm{coh}}-\Delta Q_{\mathrm{inc}}}{\Omega / \omega_{0}-\left(Q_{\mathrm{ex}}+\Delta Q_{\mathrm{inc}}\right)}\left(-\frac{a^{2}}{2} \frac{\mathrm{d} \psi_{a}}{\mathrm{~d} a}\right) \\
\times b \psi_{b}(b) \psi_{p}(p) \mathrm{d} a \mathrm{~d} b \mathrm{~d} p=1,
\end{aligned}
$$

$\psi_{a}, \psi_{b}$, and $\psi_{p}$ are the corresponding distribution functions normalized as $\int a \psi_{a} \mathrm{~d} a=1, \int b \psi_{b} \mathrm{~d} b=1$, and $\int \psi_{p} \mathrm{~d} p=$ 1. $Q_{\text {ex }}$ includes the bare tune plus tune shifts due to external nonlinearities and chromaticity effects,

$$
Q_{\mathrm{ex}}(a, b, p)=Q_{0}+\Delta Q_{\mathrm{oct}}(a, b)+\Delta Q_{\xi}(p) .
$$

The way Eq. (7) treats tune shifts of different nature has crucial consequences for the case with nonlinear space charge only. In this situation $Q_{\mathrm{ex}}$ is the bare tune and the integral has no pole which means the solution $\Omega$ is real. Thus, the dispersion relation [Eq. (7)] predicts no damping for this case even if the coherent tune overlaps the incoherent tune spectrum.

In the absence of amplitude-dependent tune shifts, Eq. (7) reduces to the usual dispersion relation (e.g., [14]) for the $\delta p$-induced linear Landau damping, which is normally written as

$$
\Delta Q \omega_{0} \int \frac{f\left(\omega_{s}\right)}{\omega_{s}-\Omega} \mathrm{d} \omega_{s}=1,
$$

here for the slow wave $\omega_{s}=n \omega_{0}-\omega_{\beta}$. This dispersion relation is often used to estimate the transverse stability due to momentum spread and chromaticity.

Here, we obtain stability diagrams from the dispersion relation [Eq. (7)] numerically by direct three-dimensional integration. The dispersion integral is calculated by introducing a small (ideally infinitesimal) imaginary part to the
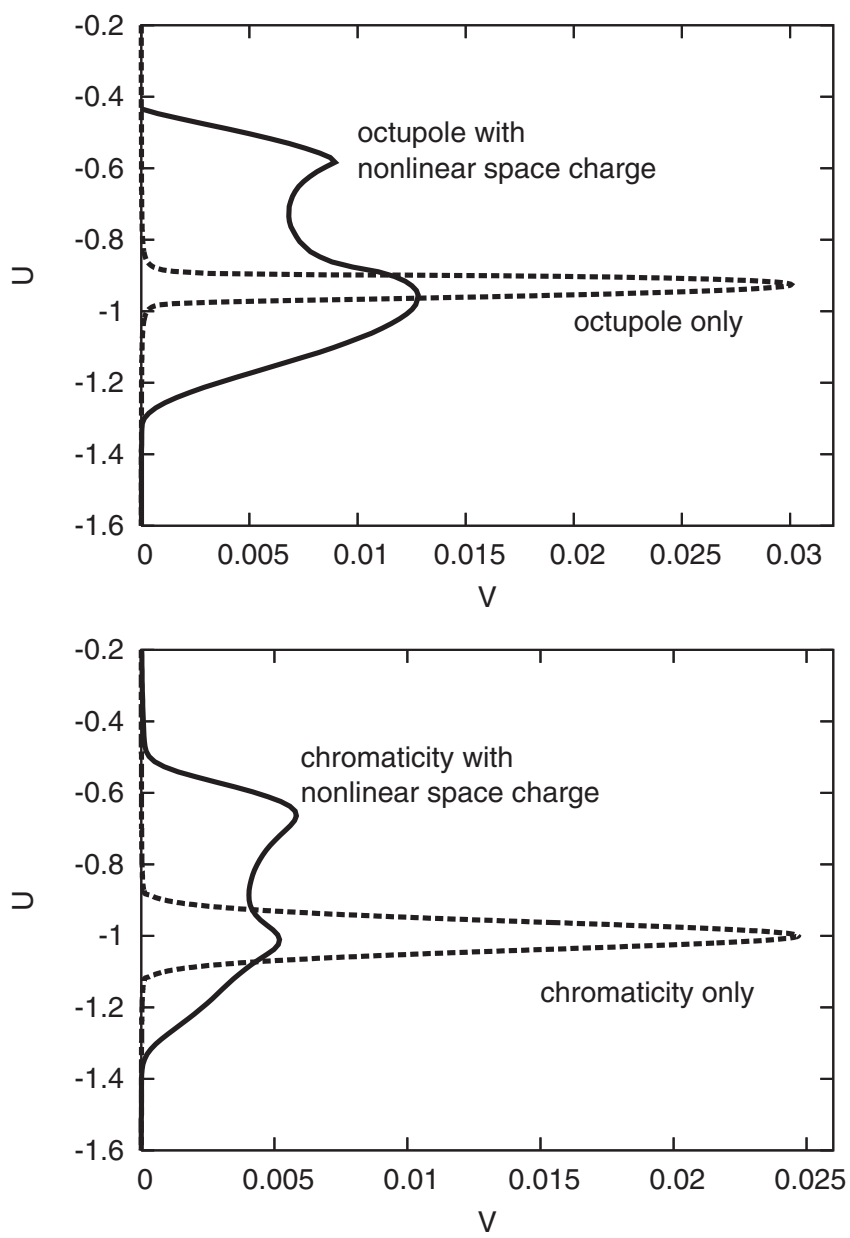

FIG. 2. Stability diagrams obtained with the dispersion relation [Eq. (7)]. Top: for an octupole only (a beam with the KV distribution, linear space charge) and for a combination of this octupole with nonlinear space charge (waterbag distribution); bottom: for the chromaticity effect only (with linear space charge) and for the combination with nonlinear space charge. $V+i U$ is the normalized impedance; for the definition see Eq. (4). 
frequency $\Omega$, with a leading sign corresponding to a positive growth rate. The amplitude-dependent incoherent tune due to space charge [the function $\kappa(a, b)$ in Eq. (3)] is estimated numerically for all individual amplitude combinations by averaging over particle trajectories, which is an approximation. The results are validated for distributions with analytic solutions. Arbitrary distributions $\psi_{a}$ and $\psi_{b}$ can be taken; in this work we only consider the waterbag and the Gaussian distribution. The change in the density distribution due to space charge is neglected. Including the external nonlinearity from Eq. (5) for $Q_{\mathrm{ex}}$, the frequency shift of the classical anharmonic oscillator can be taken into account analytically, $\Delta Q_{\text {oct }}(a)=$ $-(\beta c)^{2} /\left(16 \omega_{\beta 0} \omega_{0}\right) K_{3} a^{2}$.

Examples for the solutions of the dispersion relation [Eq. (7)] are presented in Fig. 2. These stability diagrams show the contour level for $\operatorname{Im}(\Omega)=0$ in the normalized impedance plane $V+i U$, see Eq. (4). The stable areas are the regions enclosed by the curves and by the $U$-axis. In Fig. 2 (top), the stability boundary for an octupole alone as a damping mechanism (dashed line) is presented. Linear space charge is included, which can be seen from the shift of the stability area to $U=-1$. It is compared with the stability boundary for the combination (solid line) of the same octupole with nonlinear space charge for the waterbag distribution. An octupole of the advantageous polarity is considered, the relative strength of the nonlinearities is characterized by the parameter $\chi_{\text {oct }} \approx 0.12$. Figure 2 (bottom) compares stability diagrams for the chromaticity effect only (dashed line) and for the combination (solid line) with nonlinear space charge for the waterbag distribution. The incoherent tune spreads correspond here to $\chi_{\xi} \approx 0.2$. The case of the chromaticity effect alone is also a solution of Eq. (9).

In order to characterize the stability area it is appropriate to use two approximate widths: the one along the imaginary impedance axis is $\delta U_{\text {st }}$, and along the real impedance axis we use $\delta V_{\text {st }}$. Figure 2 demonstrates that the stability parameter $\delta U_{\text {st }}$ is given by $\delta Q_{\text {oct }}$ (top plot) and by $\delta Q_{\xi}$ (bottom plot) in the cases of external effects only. For the combinations with nonlinear space charge the stability parameter $\delta U_{\mathrm{st}}$ is basically defined by $\delta Q_{\mathrm{sc}}$ for both of these examples, since space charge is the dominating effect for the tune spread considered here.

The stability diagrams shown in Fig. 2 also illustrate the space-charge induced loss of Landau damping. As it can be seen in Fig. 2, linear space charge shifts the center of the stability area downwards to $U=-1$. The coherent tune shift due to image currents is normally much smaller than the tune shift due to direct space charge. Therefore the $U$ value is usually close to 0 . For relatively weak space charge the tune shift $\delta U_{\text {st }}$ is large enough to cover $U=0$ and thus these beams are stable. As space charge becomes stronger, this is not the case anymore, and dipole perturbations grow exponentially.

\section{RESULTS}

\section{A. Nonlinear space charge only}

For the case with nonlinear space charge only, i.e., without external nonlinearities, extensive simulation scans with the code PATRIC for different $\operatorname{Re}\left(Z^{\perp}\right)$ and $\operatorname{Im}\left(Z^{\perp}\right)$ did not indicate stability for finite real impedances, which supports the prediction of the dispersion relation [Eq. (7)].

It is important to point out that for these simulations one has to exclude all the possible sources of tune spreads, besides the direct space-charge field. This implies not only exclusion of chromaticity and nonlinear lattice elements. For example, we used a round pipe as boundary condition. Other boundaries, like elliptic or rectangular ones, produce external incoherent tune shifts and a corresponding finite stability area.

As to the physical interpretation of this phenomenon, an explanation using a single-particle equation of motion can be found in [4]. Here we refer to the general conservation of momentum, which does allow a situation where the internal interactions alone affect the motion of the mass center. For transverse dipole oscillations it means that, if space charge (an intrinsic force, related to the beam center) is the single source of nonlinearity, it cannot affect the coherent frequency. However, if octupole magnets are introduced (an external nonlinearity, related to the chamber center), they cause amplitude-dependent tune shifts, which are superimposed onto the tune spread due to nonlinear space charge. Thus the interplay of the octupole cubic nonlinearity and nonlinear space charge modifies the stability diagrams.

\section{B. Octupole with nonlinear space charge}

The ratio between incoherent tune spreads induced by the external effect and by nonlinear space charge (e.g. $\left.\chi_{\text {oct }}=\delta Q_{\text {oct }} / \delta Q_{\text {sc }}\right)$ determines the stability boundary. Figure 3 illustrates this fact with dependencies of the widths of the stable area $\delta U_{\text {st }}$ and $\delta V_{\text {st }}$ for the octupole only case and for the combination of this octupole with nonlinear space charge, as is predicted by the dispersion relation [Eq. (7)]. For weak octupoles (left edges in Fig. 3), the size of the stable area along $U$ is determined by the tune spread due to nonlinear space charge $\delta Q_{\text {sc }}$. Meanwhile, the stability parameter $\delta V_{\text {st }}$ is proportional to the octupole strength $K_{3}$. Our example in Fig. 2 corresponds to this regime. For strong octupoles (right edges in Fig. 3), $\delta Q_{\text {oct }} \gg \delta Q_{\text {sc }}$ and hence stability properties are dominated by the octupole.

The strength of the octupole lenses, which are used to cure instabilities, is restricted by the requirements for the dynamic aperture. In our numeric simulations we consider the case of a relatively weak octupole, which corresponds to the conditions in the FAIR synchrotrons. This gives also a numeric advantage, since for a weak octupole one can assume a large beam intensity to make growth and damp- 

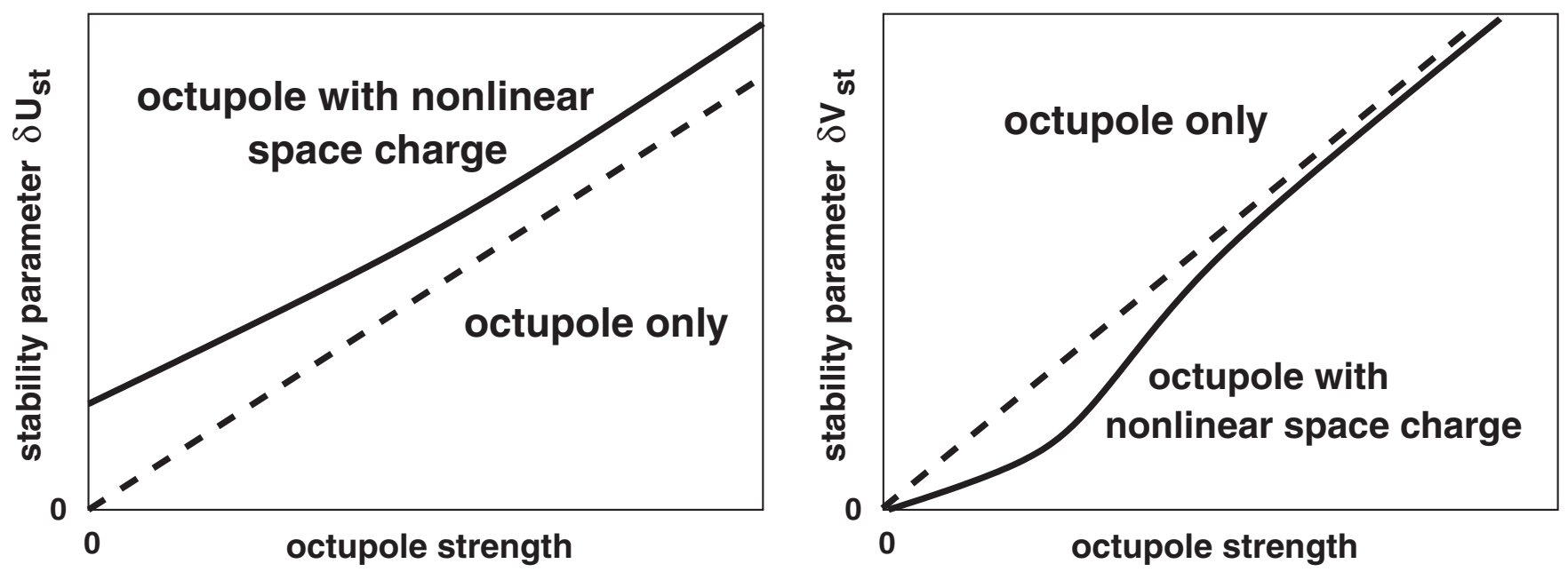

FIG. 3. Illustration for different regimes of stability provided by an octupole and nonlinear space charge: widths of the stability area along $\operatorname{Im}\left(Z^{\perp}\right)$ (left) and along $\operatorname{Re}\left(Z^{\perp}\right)$ (right) in a dependence from the strength of the octupole, with the tune spread due to space charge staying constant.

ing times smaller, which ensures reasonable computing times.

In order to compare the predictions of the dispersion relation [Eq. (7)] with PIC simulations for the interplay of an octupole with nonlinear space charge, we consider the same conditions as those assumed to obtain results in Fig. 2, i.e., a transverse waterbag distribution and $\chi_{\text {oct }} \approx$ 0.12 . First, we examined the case without nonlinear space charge (as for the dashed line in Fig. 2) and found a good

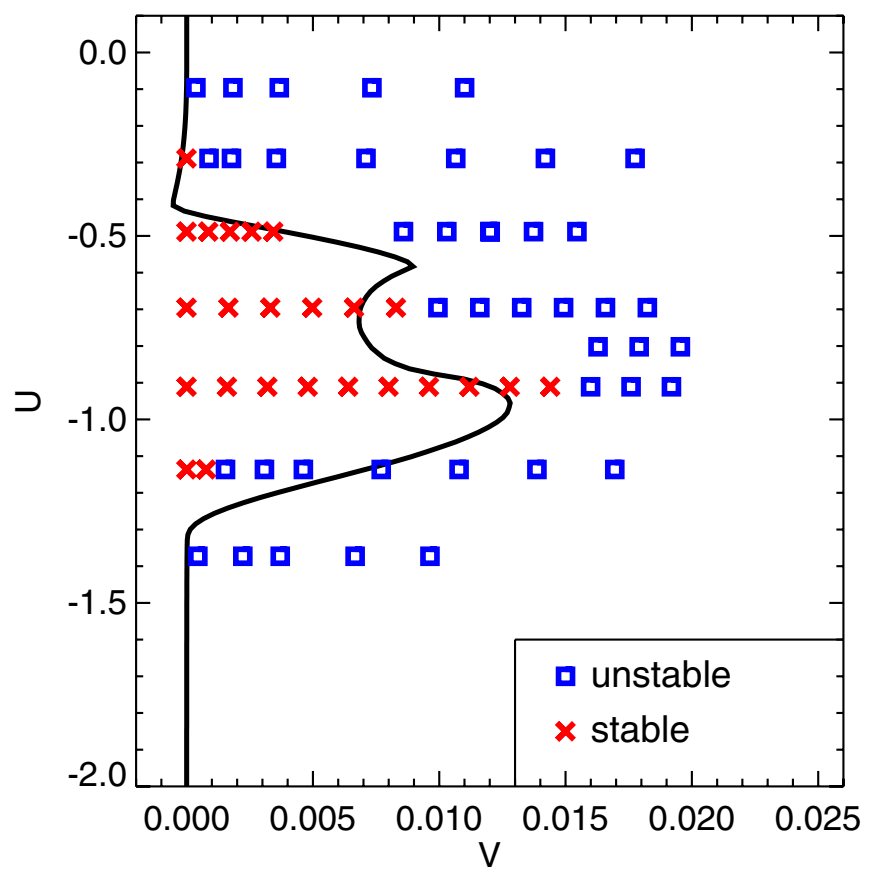

agreement between PATRIC simulations and predictions of Eq. (7).

Results of the comparison for the case with nonlinear space charge are presented in Fig. 4, left. The curve is the stability boundary from Fig. 2; the simulation scans are shown with squares (unstable) and crosses (stable) where each of these symbols is the outcome of a different simulation run. The octupole has the advantageous polarity here; the electric field is calculated self-consistently. PATRIC simulations confirm the enlargement of the stability

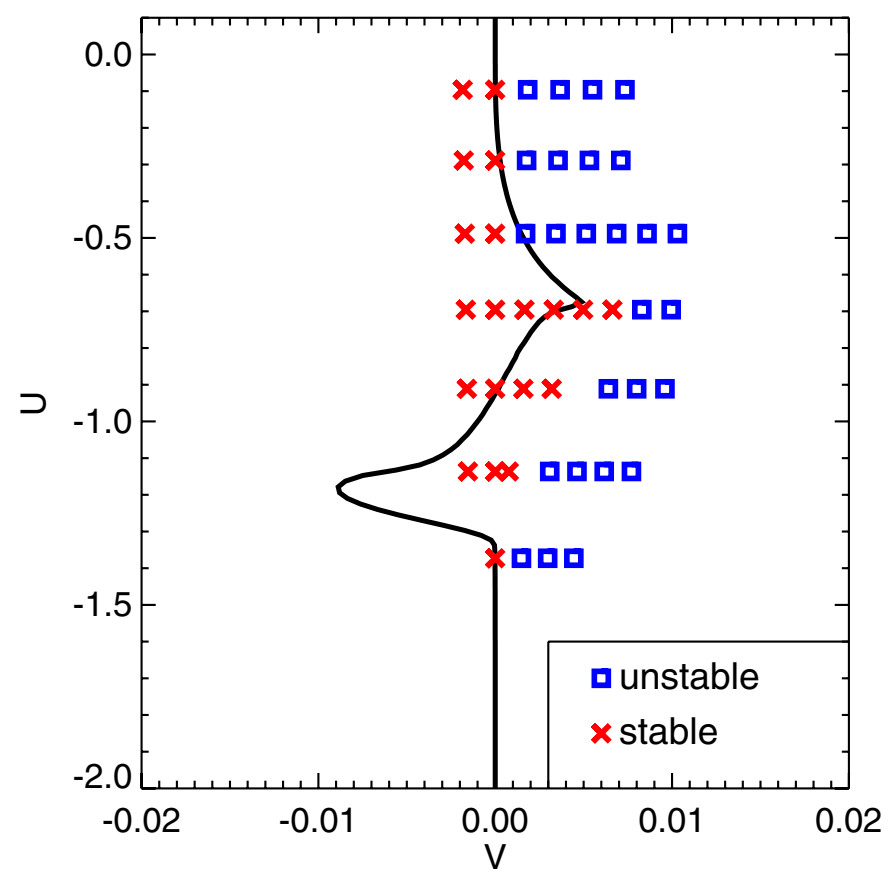

FIG. 4. (Color) Combination of nonlinear space-charge and an octupole with the advantageous polarity (left), disadvantageous polarity (right); simulations are made with self-consistent electric field, transverse waterbag distribution. The symbols are the results of simulations; the lines are stability boundaries from the dispersion relation. 
along $\operatorname{Im}\left(Z^{\perp}\right)$ (regarding the case with an octupole only) and the extent of the stability area in $\operatorname{Re}\left(Z^{\perp}\right)$, as it is predicted by the dispersion relation [Eq. (7)].

For the case of an octupole with the disadvantageous polarity the dispersion relation [Eq. (7)] yields the stability boundary which is presented in Fig. 4, right. Here, an octupole with the same modulus strength $\left|K_{3}\right|$, but with the opposite polarity with respect to Fig. 4 (left) is assumed. A comparison between the plots in Fig. 4 shows that the usage of an octupole with the disadvantageous polarity substantially reduces the stability area along the $\operatorname{Re}\left(Z^{\perp}\right)$ axis. Part of the stability boundary is located at negative $V$ here, which means a so-called "antidamping" in this region. This implies instabilities for zero and negative $\operatorname{Re}\left(Z^{\perp}\right)$ (also discussed in [9]). Figure 4 (right) also presents results of our PATRIC simulations, which support the reduction of the stability. However, no antidamping was observed in these simulations with a self-consistent electric field.

\section{Simulations with frozen space charge}

Facing the necessity of complete 3D simulations for studies of the long-term beam stability with constraints of limited computing time and CPU power, the question is often raised, whether simulations with non-selfconsistent space charge are accurate enough. Here, we examine this question by comparing simulations using self-consistent space-charge with those using frozen space-charge for the case of an octupole and nonlinear space charge.

Figure 5 presents results of PATRIC simulations using frozen space charge obtained for identical parameters as those shown in Fig. 4. Considering the case of an octupole with the advantageous polarity, we see that the simulations for frozen space charge agree with the self-consistent model in $\delta U_{\text {st }}$ (width of the stability area along $U$ ) but disagree in $\operatorname{Re}\left(Z^{\perp}\right)$-thresholds. For an octupole with the disadvantageous polarity, our simulations with frozen space charge demonstrated antidamping (triangles in Fig. 5, right). In the self-consistent simulations antidamping did not occur. Therefore antidamping can be related to the non-self-consistent treatment of nonlinear space charge in the simulations and also in the dispersion relation.

\section{Stability with a Gaussian distribution}

In the present section we discuss the results obtained for a transverse Gaussian distribution. Because of the stronger incoherent tune spread for the same rms beam radius, the dispersion relation predicts a larger stability area compared to the waterbag distribution, see the stability diagram (line) in Fig. 6. Here, the same octupole coefficient is assumed as for Fig. 4, left.

In Fig. 6 the results of PATRIC simulations are represented as symbols. In contrast to the waterbag distribution, we find strong discrepancies in the extension of the stability area obtained from the self-consistent simulations and
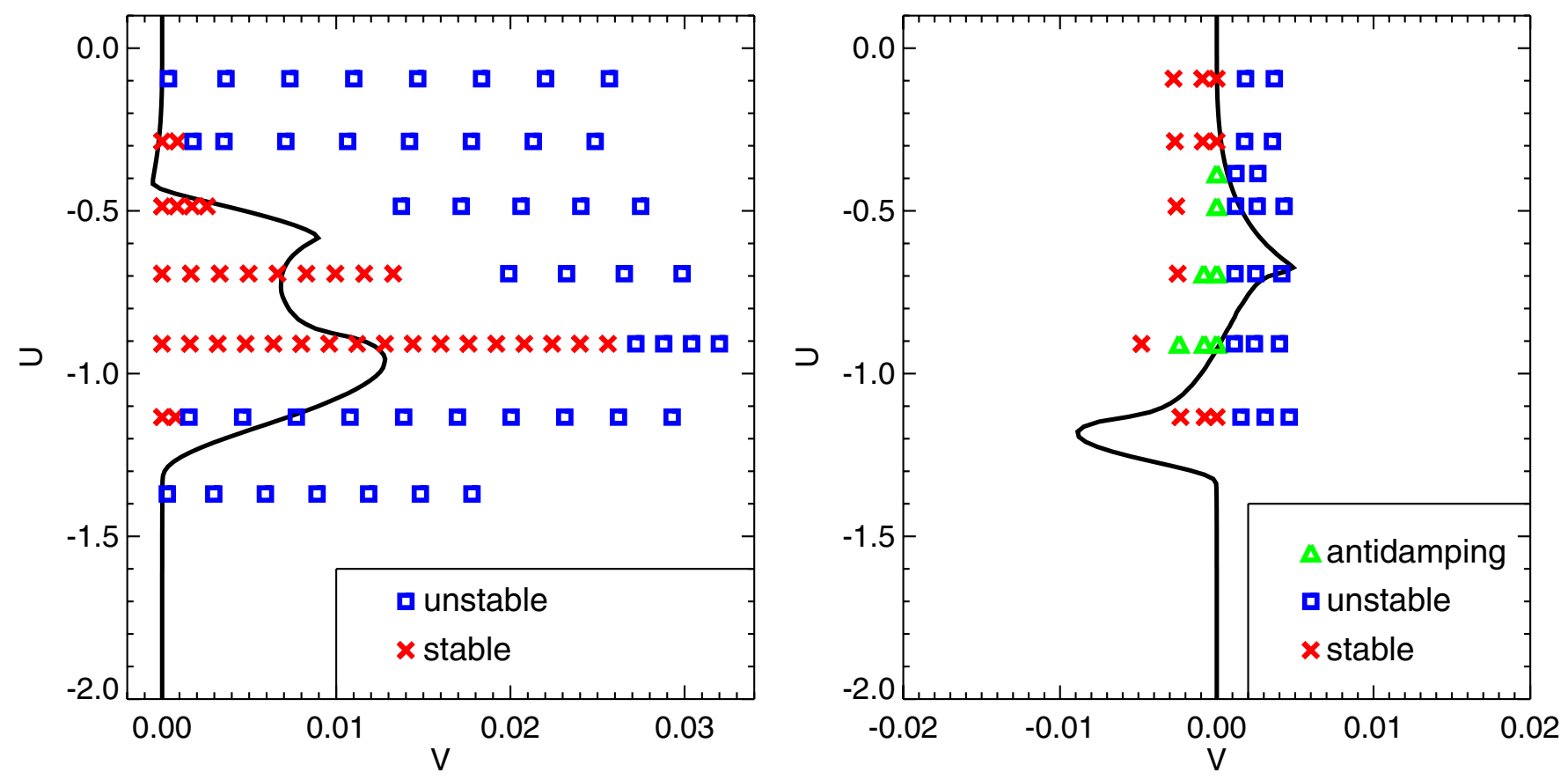

FIG. 5. (Color) Combination of nonlinear space-charge and an octupole with the advantageous polarity (left), disadvantageous polarity (right); simulations are made with the frozen electric field for a waterbag distribution. The symbols are the results of simulation runs; the lines are stability boundaries from the dispersion relation. 

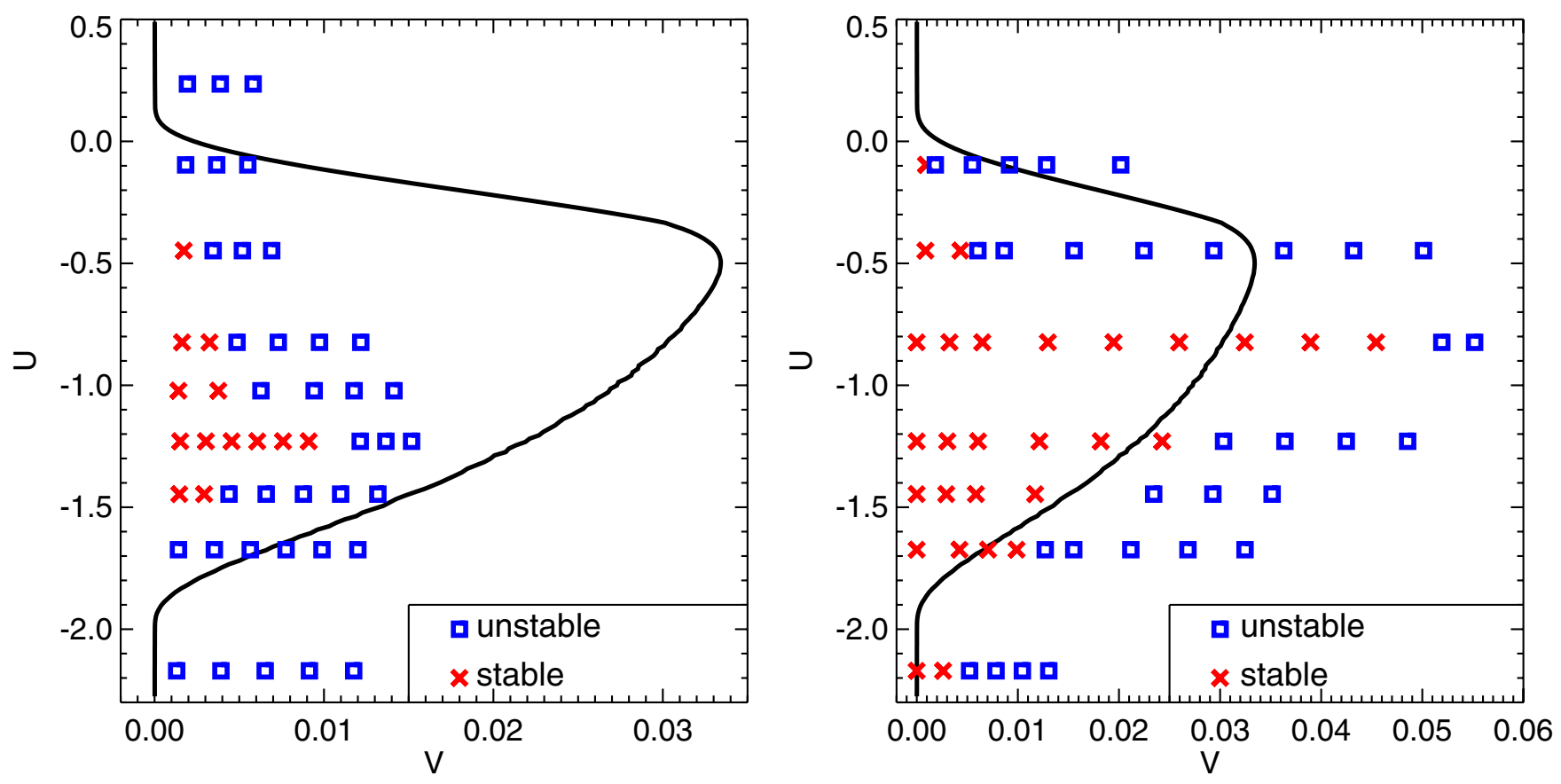

FIG. 6. (Color) Combination of nonlinear space-charge and an octupole with the advantageous polarity, Gaussian distribution; left: simulations with self-consistent space charge; right: simulated with frozen electric field. Symbols are results of simulation runs; lines are stability diagrams from the dispersion relation.

from the dispersion relation (see Fig. 6, left). The width of the stability area represented by $\delta U_{\text {st }}$ and $\delta V_{\text {st }}$ obtained from the simulations is much smaller than that given by the dispersion relation. The reason for this disagreement is the strong modification of the beam distribution during the initial phase of the instability. Contrary to simulations with a waterbag distribution, the initial Gaussian beam profile starts to experience strong deformations already at the beginning of the instability evolution when the amplitude of the $\bar{x}$-oscillations is still small. The unstable mode
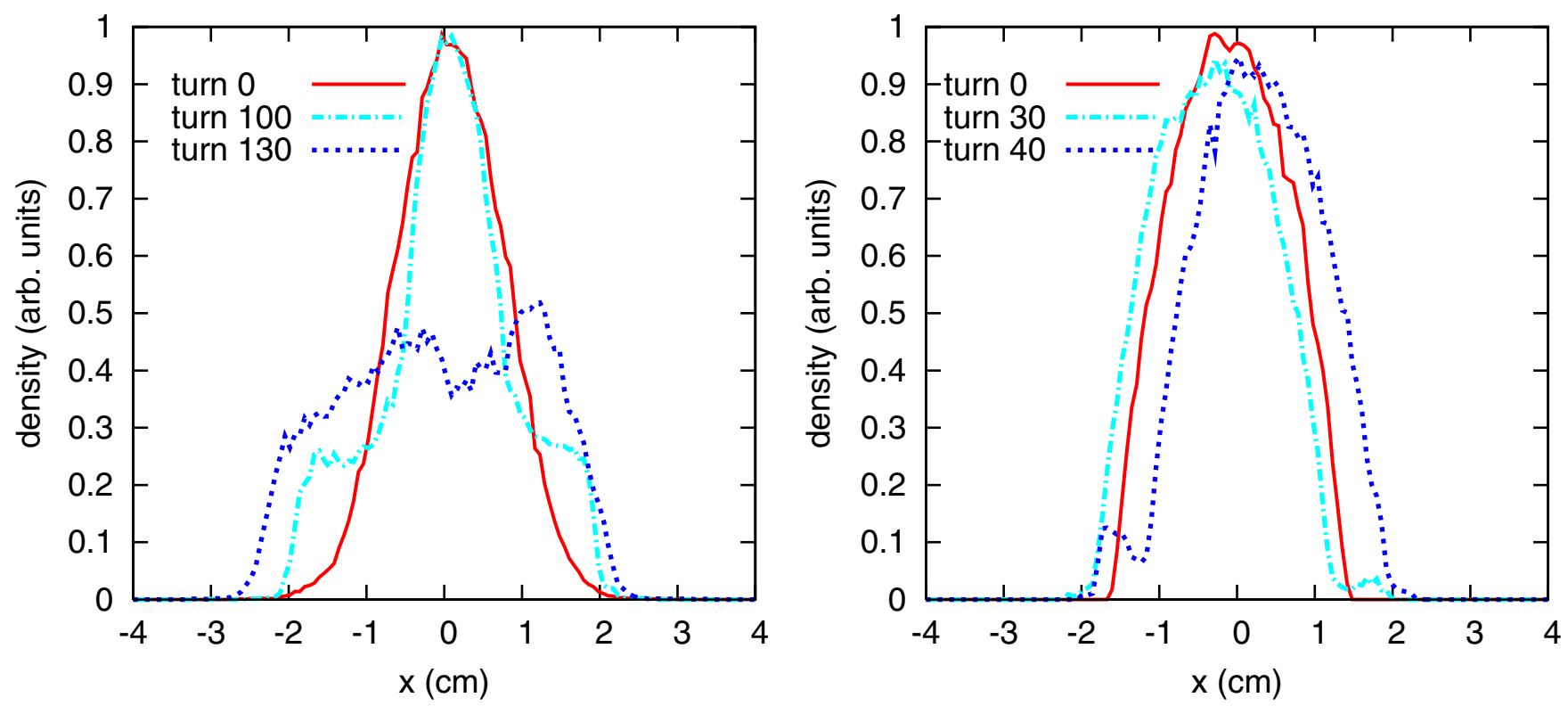

FIG. 7. (Color) Evolution of the beam profile with time for simulation runs made with self-consistent space charge. Left: Gaussian distribution, $V=0.01, U=-0.82$, see Fig. 6 (left). Right: waterbag distribution, $V=0.012, U=-0.7$, see Fig. 4 (left). 
cannot be described as a rigid dipole mode and radial mode coupling is important. The beam profile becomes flatter which causes a smaller space-charge tune spread and reduces the stability. With an initial waterbag distribution the beam profile does not deform during the exponential growth phase of the unstable oscillations. An example is shown in Fig. 7. The plot on the left-hand side presents the development of the instability in the case of an initial Gaussian distribution which would be stable according to the dispersion relation. Figure 7 (right) shows the beam profile for an unstable waterbag distribution. Here the beam profile stays rigid until the amplitude of $\bar{x}$ becomes large.

Simulations performed with frozen space charge provide a different conclusion: the stability area (see Fig. 6, right) is in a reasonable agreement, with a somewhat larger $\delta V_{\mathrm{st}}$, compared to the prediction of the dispersion relation. The beam oscillations, both stable and unstable, evolve in this case without significant profile modifications.

Results of the simulations scans for a Gaussian distribution and the comparisons between dispersion relation and simulations suggest that radial mode coupling and nonlinear dynamics can strongly change the stability properties. For a better understanding, more detailed analytical as well as simulation studies are necessary.

\section{E. Chromaticity with nonlinear space charge}

Landau damping due to chromaticity and momentum spread plays an important role for the beam stability considerations in accelerator rings. As it was already mentioned above (see Fig. 2, bottom), the dispersion relation [Eq. (7)] suggests that inclusion of nonlinearity due to space charge can significantly modify the stability properties. While linear space charge only shifts the stability diagram along $\operatorname{Im}\left(Z^{\perp}\right)$ (dashed line in Fig. 2, bottom), nonlinear space charge strongly changes both of the stability parameters $\delta U_{\text {st }}$ and $\delta V_{\text {st }}$ (solid line in Fig. 2, bottom).

Similarly to our discussion in Sec. V B, there are different regimes of stability according to the ratio between tune spreads due to chromaticity and due to nonlinear space charge. The influence of nonlinear space charge is especially pronounced in the regime where its tune spread is dominating, $\delta Q_{\mathrm{sc}} \gg \delta Q_{\xi}$. This regime will be considered in the following.

An important difference between predictions of the dispersion relation with and without nonlinear space charge can be clearly seen if we consider a scaling momentum spread. Figure 8 presents stability diagrams obtained from Eq. (7) for two $\delta p / p_{0}$, where the second one (dashed lines) is twice as large as the first one (solid lines). The tune spreads correspond to $\chi_{\xi} \approx 0.11$ for the solid lines and $\chi_{\xi} \approx 0.22$ for the dashed lines; for the top plot in Fig. 8 $\delta Q_{\text {sc }}=0$. Without nonlinear space charge the stability area is doubled as a result of the $\delta p$ increase, see Fig. 8 top. In contrast, if the amplitude-dependent tune shift for the waterbag distribution is taken into account, $\delta U_{\text {st }}$ nearly does not change and $\delta V_{\text {st }}$ is enlarged with a factor of approximately 3 , see Fig. 8, bottom.

Results of our simulation scans with the PATRIC code are presented in Fig. 9; in the right-hand side plot the momentum spread is twice as large as in the one on the left-hand side. Consistent with our figures above, the blue squares indicate instability whereas red crosses correspond to a stable beam. The simulations are made with self-consistent electric field and with an initial transverse waterbag distribution. The stability boundaries obtained from the dispersion relation [Eq. (7)] for the combination of the chromaticity with nonlinear space charge are shown in Fig. 9 as lines. The simulation scans confirm fairly well the results from the dispersion relation concerning the scaling of the stability area. We observe that $\delta U_{\text {st }}$ is nearly the same for these two parameter sets, while $\delta V_{\text {st }}$ is approximately 3 times larger for the doubled momentum spread. These results are in strong disagreement with predictions of a dispersion relation which does not take into account nonlinear space charge. The quantitative comparison between the simulation results and the dispersion relation [Eq. (7)] show that there is a good agreement in the extent of the stability area in $\operatorname{Re}\left(Z^{\perp}\right)$, but for $\delta U_{\text {st }}$ our PATRIC simulations reveal only approximately half of the width as given by the dispersion relation.
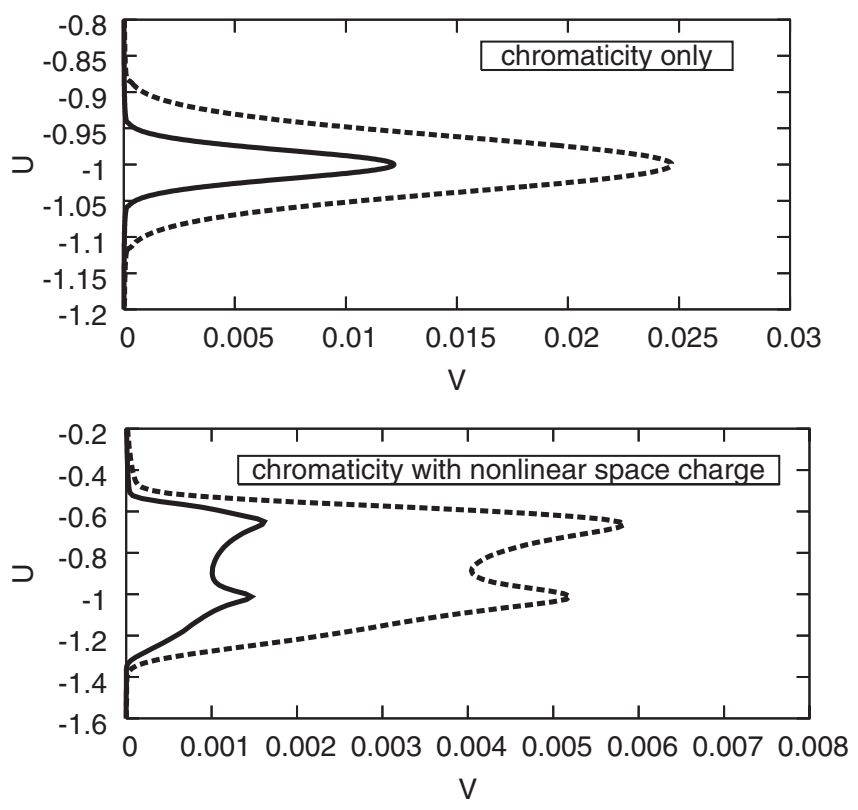

FIG. 8. Stability due to chromaticity effect as given by the dispersion relation [Eq. (7)], for dashed lines the momentum spread is doubled regarding to solid lines. Top plot: chromaticity only (with linear space charge); bottom plot: chromaticity effect taking into account nonlinear space charge for waterbag distribution. Note different axis scales. 

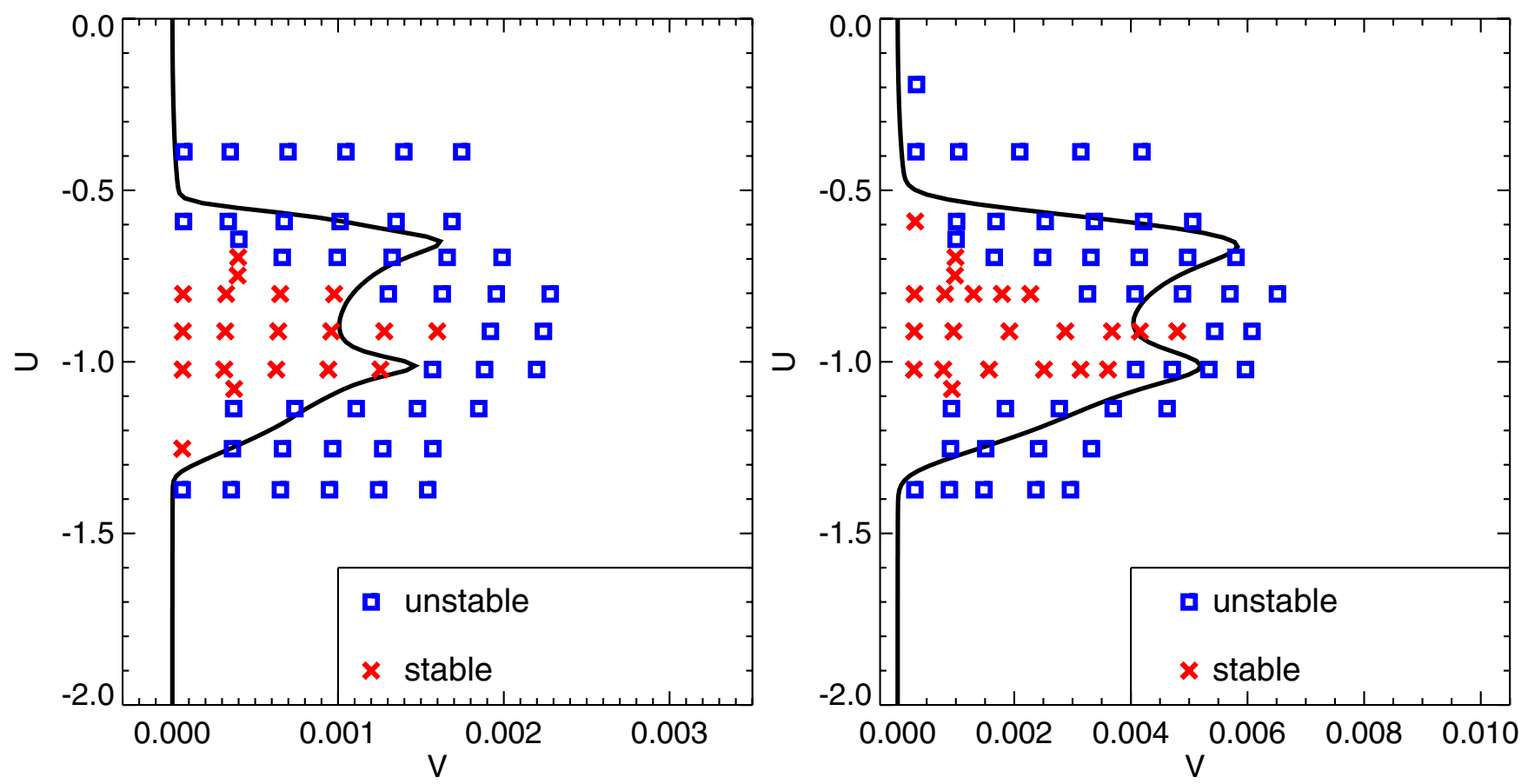

FIG. 9. (Color) Combination of chromatic effects and nonlinear space-charge; in the right plot the momentum spread is doubled in comparison with the left plot. The symbols are the results of simulation runs; lines are stability boundaries from the dispersion relation.

\section{CONCLUSIONS}

Particle tracking simulations have been performed to investigate the stability properties of coasting beams in the transverse plane. The effects of nonlinear space charge, octupoles, and chromaticity have been examined as single effects and in combinations. These effects are crucial for beam stability and are additional to linear space charge, which causes the loss of Landau damping by shifting the incoherent betatron spectrum from the coherent line (on a stability diagram, a shift of the stability area downwards). A dispersion relation based on Ref. [8] with twodimensional tune spread allows one to take into account all these effects. The dispersion relation has been solved and used as a guideline for comparisons with simulation results. Beam parameters corresponding to relatively strong space-charge effects compared to the octupole or chromaticity induced effects were chosen.

In the case of nonlinear space charge only, without octupoles or chromaticity, our simulation scans confirm the prediction of the dispersion relation [Eq. (7)]. Nonlinear space charge alone does not provide Landau damping even if the coherent frequency overlaps the tune spread induced by nonlinear space charge.

The interplay of an octupole with nonlinear space charge strongly modifies the stability properties, especially for a weak octupole (which induces smaller tune spreads than that due to nonlinear space charge). The width of the stability area along the imaginary impedance axis $\delta U_{\mathrm{st}}$ is determined by the tune spread caused by nonlinear space charge, while the extent of the stability area in $\operatorname{Re}\left(Z^{\perp}\right)$ depends on the octupole strength. For weak space charge the stability area is determined by the octupole. Our PATRIC simulation scans confirm these conclusions.

Simulations with self-consistent electric field confirm the prediction of the dispersion relation, that using an octupole with the disadvantageous polarity substantially reduces the stability area by decreasing the stability thresholds in $\operatorname{Re}\left(Z^{\perp}\right)$. However, no antidamping [instabilities for zero and negative $\operatorname{Re}\left(Z^{\perp}\right)$ ], which is predicted by the dispersion relation, was observed in self-consistent simulations.

The question of the applicability of frozen space charge (the shape of the space-charge field relative to the beam center is kept constant) for stability simulations was addressed. For an octupole with the advantageous polarity, the results obtained with a frozen electric field agree with the self-consistent model in the width of the stability area $\delta U_{\text {st }}$ but disagree quantitatively in the $\operatorname{Re}\left(Z^{\perp}\right)$-thresholds. For an octupole with the disadvantageous polarity, antidamping was observed in simulations with frozen space charge, in contrast to the results obtained with selfconsistent space charge. From this we conclude that antidamping can be related to the non-self-consistent treatment of nonlinear space charge in the simulations and also in the dispersion relation. Therefore frozen space charge has to be used with caution in simulation studies of collective effects.

A transverse Gaussian distribution induces stronger space-charge tune shifts than the rms equivalent waterbag distribution does. Hence, the dispersion relation predicts larger stability parameters $\delta U_{\text {st }}$ and $\delta V_{\text {st }}$. However, PIC 
simulations for the Gaussian distribution with selfconsistent space charge provide stability thresholds which are approximately a factor 3 smaller than those given by the dispersion relation (which may be specific for beam parameters assumed). A possible reason is radial mode coupling and the associated deviations from a rigid dipole mode. For a better understanding, more detailed analytical as well as simulation studies are necessary.

Landau damping due to chromaticity, which often plays a central role in the transverse stability analysis, is also strongly modified by the inclusion of nonlinear space charge. The dispersion relation [Eq. (7)] predicts not only significant changes in stability parameters $\delta U_{\text {st }}$ and $\delta V_{\text {st }}$, but also a different scalings of these parameters for an increasing momentum spread (or chromaticity). Simulation scans with the code PATRIC confirm these predictions of the dispersion relation concerning relative changes of the stability area. Quantitative comparisons show that there is a good agreement in the extent of the stability area in $\operatorname{Re}\left(Z^{\perp}\right)$, but along the imaginary impedance axis our PIC simulations give approximately 2 times smaller $\delta U_{\text {st }}$ than that from the dispersion relation.

\section{ACKNOWLEDGMENTS}

This work was supported by EU design study (Contract No. 515873 DIRACsecondary-Beams).
[1] FAIR-Baseline Technical Report, 2006: http:// www.gsi.de/fair/reports/btr.html.

[2] K. Y. Ng, FERMILAB-FN-0760-AD, 2004.

[3] O. Boine-Frankenheim, I. Hofmann, and V. Kornilov, Proceedings of EPAC 2006, Edinburgh, UK, 2006, p. 1882.

[4] D. Möhl and H. Schönauer, Proceedings of the IX International Conference on High Energy Accelerators, Stanford, 1974, p. 380.

[5] G. Merle and D. Möhl, CERN/ISR/300/GS/69-66, 1969.

[6] J.S. Berg and F. Ruggiero, CERN SL-AP-96-71, 1996.

[7] E. Métral and F. Ruggiero, Proceedings of EPAC2004, Lucerne, Switzerland, 2004, p. 1897.

[8] D. Möhl, CERN/PS 95-08 (DI), 1995.

[9] D. V. Pestrikov, Nucl. Instrum. Methods Phys. Res., Sect. A 562, 65 (2006).

[10] M. Blaskiewicz, Phys. Rev. ST Accel. Beams 4, 044202 (2001).

[11] V. Balbekov, Phys. Rev. ST Accel. Beams 9, 064401 (2006); FERMILAB-FN-0782-AD, 2006.

[12] O. Boine-Frankenheim and V. Kornilov. Proceedings of ICAP2006, Chamonix Mont-Blanc, 2006.

[13] Ming-Chih Lai, Numer. Methods Partial Differ. Equ. 20, 72 (2004).

[14] A. Hofmann, Landau Damping, CERN Accelerator School, Rhodes, Greece, 1993 (CERN 95-06 v.1, p. 275). 